\title{
Retinal blood flow in critical illness and systemic disease: a review
}

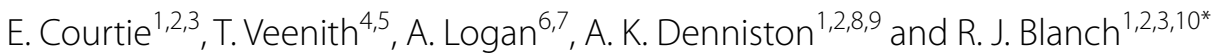

\begin{abstract}
Background: Assessment and maintenance of end-organ perfusion are key to resuscitation in critical illness, although there are limited direct methods or proxy measures to assess cerebral perfusion. Novel non-invasive methods of monitoring microcirculation in critically ill patients offer the potential for real-time updates to improve patient outcomes.

Main body: Parallel mechanisms autoregulate retinal and cerebral microcirculation to maintain blood flow to meet metabolic demands across a range of perfusion pressures. Cerebral blood flow (CBF) is reduced and autoregulation impaired in sepsis, but current methods to image CBF do not reproducibly assess the microcirculation. Peripheral microcirculatory blood flow may be imaged in sublingual and conjunctival mucosa and is impaired in sepsis. Retinal microcirculation can be directly imaged by optical coherence tomography angiography (OCTA) during perfusiondeficit states such as sepsis, and other systemic haemodynamic disturbances such as acute coronary syndrome, and systemic inflammatory conditions such as inflammatory bowel disease.

Conclusion: Monitoring microcirculatory flow offers the potential to enhance monitoring in the care of critically ill patients, and imaging retinal blood flow during critical illness offers a potential biomarker for cerebral microcirculatory perfusion.
\end{abstract}

Keywords: Critical illness, Retinal blood flow, Optical coherence tomography angiography

\section{Introduction}

Critical illness with multiple organ dysfunction is characterised by complex physiological and metabolic responses requiring support and optimisation of organ systems in the intensive treatment unit (ITU) [1]. Common aetiologies include sepsis $(60 \%)$, trauma, and perioperative care. Sepsis is a systemic inflammatory response to infection, mediated by the pathogen and host factors, ultimately causing multiple organ failure [2], and is a growing global concern with an estimated 48.9 million incident cases recorded worldwide in 2017, 11 million of which were fatal [3]. Septic shock describes a profound haemodynamic alteration associated with organ dysfunction,

\footnotetext{
*Correspondence: r.j.blanch@bham.ac.uk

${ }^{1}$ Neuroscience and Ophthalmology, Institute of Inflammation and Ageing, College of Medical and Dental Sciences, University of Birmingham, Birmingham, UK

Full list of author information is available at the end of the article
}

including hypovolaemia and myocardial depression [4]. Early diagnosis of sepsis and prompt treatment to reduce multiple organ failure reduces mortality [5], but survivors often have physical and neurocognitive disability referred to as post-intensive care syndrome (PICS) [6]. Attempts to improve perfusion and end-organ microcirculation using inotropes and fluids have produced variable results [7].

Microcirculation facilitates tissue oxygenation and the exchange of substances between tissues and blood. In septic shock, physiological haemodynamic parameters, such as mean arterial pressure (MAP), may not be indicative of microcirculatory perfusion [8]. Patients with sepsis often have microcirculatory alterations, such as reduced functional capillary density, which reduces oxygen delivery to vital organs and plays a key role in the development of organ dysfunction $[4,9,10]$. While the extent of these microcirculatory alterations in the brain is 
less well characterised than in other organs, post-mortem examination of septic patients demonstrated multiple small ischaemic lesions, suggesting microvascular insufficiency [11]. Sepsis-associated brain dysfunction (SABD) is a common sepsis-related organ dysfunction [12], and probably involves reduced cerebral blood flow (CBF) causing cerebral ischaemia [12]. Compromised cerebral blood supply often causes both immediate and delayed irreversible damage with associated neurocognitive decline and poor outcome [13]. It is, therefore, essential to be able to monitor CBF during critical illness.

The retina and brain share similar microvascular anatomy, and while direct visualisation of CBF is difficult, retinal imaging is comparatively convenient [14]. Retinal structural and blood flow changes associated with systemic and central nervous system illness are increasingly reported [15-17] with the use of ocular imaging to assess systemic disease termed "oculomics" [18]. Retinal changes may, therefore, associate with CBF in critically ill patients, offering a novel biomarker to monitor in realtime and reduce cerebral hypoperfusion.

This review discusses the relationship between cerebral and retinal blood flow, and the relevance of that relationship to systemic pathology and monitoring microcirculatory perfusion in critical illness, focussing more on sepsis.

\section{Cerebral and retinal blood flow autoregulation Cerebral blood flow autoregulation}

The human brain consumes $20 \%$ of the body's energy at rest, dependent on CBF to ensure the delivery of oxygen, nutrients and removal of metabolic waste products [19]. Global or focal hypoperfusion rapidly results in brain damage.

Under normal physiological conditions, blood flow to the brain remains constant, in part due to the contribution of large arteries to vascular resistance, but also because of autoregulation [20]. CBF autoregulation is the ability of the brain to maintain relatively constant blood flow despite changes in perfusion pressure while matching flow to local metabolic demand [20]. Cerebral perfusion pressure (CPP) is determined by MAP and intracranial pressure (ICP), where autoregulation adjusts vascular resistance to maintain $\mathrm{CBF}$. CBF autoregulation is complex, with multiple proposed overlapping regulatory mechanisms, including myogenic, neurogenic, metabolic and endothelial regulation [21]. Most data suggest reduced $\mathrm{CBF}$ and impaired $\mathrm{CBF}$ autoregulation in sepsis [22].

\section{Cerebral microcirculation}

The cerebral microcirculation is the driver of oxygen transport and waste removal in the cortex [23], supplied by the penetrating arteriolar network from the brain surface. Every neurone in the brain is within $20 \mu \mathrm{m}$ of a capillary [24], receiving oxygen and nutrients yet remaining protected from fluctuations in plasma composition, circulating proteins and immune cells by the blood-brain barrier (BBB). Endothelial cells (EC) and their tight cell junctions are the fundamental constituents of the $\mathrm{BBB}$ and regulate paracellular transport [24].

The neurovascular unit is in part responsible for the coupling of blood flow with brain activity and is made up of EC, pericytes, astrocyte end-feet and vasoregulatory nerve terminals [25]. Pericytes project stellate, finger-like processes that ensheath the capillary wall [26] and contract or dilate in response to vasoactive mediators, such as nitric oxide (NO). NO is produced by neuronal nitric oxide synthase (nNOS) or neural pathways [27] to alter capillary diameter in autoregulation, shown in vivo in rat retina and ex vivo in cerebellar slice cultures [28]. This neurovascular coupling is impaired in the early stages of sepsis [29]. EC regulate CBF through the production of vasodilatory factors, including $\mathrm{NO}$ and vasoconstrictors such as endothelins, which bind to $\mathrm{ET}_{\mathrm{A}}$ receptors in the cerebrovascular smooth muscle, although endothelins also have vasodilatory effects when binding to $\mathrm{ET}_{\mathrm{B}}$ receptors on EC themselves [21].

\section{Retinal microcirculation}

The retinal vascular beds, derived from the central retinal artery, include the radial peripapillary capillary plexus (RPCP) in the nerve fibre layer, the superficial vascular plexus (SVP) spanning the ganglion cell layer (GCL) and inner plexiform layer, the intermediate capillary plexus (ICP) sitting between the inner plexiform layer and inner nuclear layer, and the deep capillary plexus (DCP) spanning the inner nuclear layer and outer plexiform layer [30]. These supply the inner retina, including the retinal ganglion cells, while the outer retina derives oxygenation and nutrition from the choriocapillaris of the choroid (Fig. 1) [31]. Campbell et al. propose OCTA nomenclature as the RPCP and SVP be grouped into the superficial vascular complex (SVC), with the ICP and DCP grouped into the deep vascular complex (DVC) to highlight anatomic location of the $\mathrm{ICP}$ at the inner plexiform/inner neuronal layer interface [30].

The foveola centralis is a depressed, avascular area of the macula, also referred to as the foveal avascular zone (FAZ). It is this area which allows the most distinct vision because of the high cone density and absence of blood vessels [31]. The circulation is particularly vulnerable in the FAZ, as the absence of retinal blood vessels leaves the cones completely dependent upon oxygen and nutrient delivery from the underlying choriocapillaris [31]. The 
FAZ is therefore highly sensitive to ischaemic events and because of this, can act as an indicator of several pathological processes [32]. Enlargement of the FAZ area has been associated with ischaemia in diabetic retinopathy and retinal vein occlusion [32].

The internal carotid artery gives rise to the ophthalmic artery, from which the central retinal artery arises [33], entering the optic nerve $(\mathrm{ON}) \quad 10-12 \mathrm{~mm}$ behind the globe [33]. The choriocapillaris is derived from the short posterior ciliary arteries, which also branch off the ophthalmic artery. The conjunctiva covers the sclera and lines the inside of the eyelids, and is also supplied by the ophthalmic artery [34].

\section{Retinal blood flow autoregulation}

Similar to CBF, retinal blood flow depends on the balance between perfusion pressure in the ophthalmic artery and the resistance of the retinal vascular bed, and is autoregulated to mirror cerebral perfusion in healthy individuals $[35,36]$. The retina has the highest density of microvascular pericytes in the body [36,37], contributing to the myogenic vascular autoregulation of blood flow and providing structural support to blood vessels [36]. Changes in ocular perfusion pressure and altered metabolic demand initiate an autoregulatory response [38], maintaining retinal but not choroidal or conjunctival blood flow [35].

Retinal circulation lacks autonomic innervation [39] and is dependent on local vasogenic factors acting on the neurovascular unit $[39,40]$. Despite the absence of sympathetic activation, retinal blood flow is able to remain constant over a range of intraocular pressures (IOP), which naturally fluctuates in daily life [38], although an elevated IOP above $40 \mathrm{mmHg}$ reduces retinal blood flow [41]. Local metabolic factors mediating retinal autoregulation include endothelin-1 which is secreted by EC and acts as a vasoconstrictor, affecting retinal vascular endothelium, pericytes and the choroid [39]. The bloodretina barrier $(\mathrm{BRB})$ has a similar structure to the $\mathrm{BBB}$ and protects retinal neurones from fluctuating plasma composition [42].

\section{Assessment of cerebral blood flow in sepsis}

Functional imaging techniques to assess CBF in realtime include direct methods: triple oxygen $\left({ }^{15} \mathrm{O}\right)$ positron emission tomography $\left({ }^{15} \mathrm{OPET}\right)$, single-photon emission computed tomography (SPECT), and magnetic resonance angiography (MRA); and indirect methods: computed tomography perfusion (CTP), functional magnetic resonance imaging (fMRI) and near-infrared spectroscopy (NIRS) [43-45]. ${ }^{15}$ OPET and SPECT use isotopes which are expensive, timeconsuming, and expose the patient to radiation. MRA is also expensive and time-consuming, and has poor temporal resolution. fMRI assesses regional variation in the ratio of oxy- to deoxyhaemoglobin, which associate with local changes in CBF, but is primarily sensitive to venous blood flow [44]. However, use of these imaging modalities to assess CBF in septic patients has not yet been reported. Further, these techniques require the transfer of the patient to a radiology unit [46], and the transfer of critically ill patients exposes them to increased risk of deterioration [47].

NIRS monitors cerebral cortical arterial, venous and capillary oxygenation at the capillary level, assessing fluctuations in microcirculatory CBF $[45,48]$. However, there is considerable variation in vessel measurements between patients, and measurements are attenuated by pigmented hair and skin because melanin attenuates light transmission [48]. A reduction in NIRS signal in patients with sepsis in the Emergency Department was associated with mortality [49]. However, the ability to differentiate between clinical outcome groups of interest was limited by variability [49].

Transcranial Doppler ultrasound (TDU) is a noninvasive, fast, real-time technique that uses the Doppler effect to assess moving red blood cells (RBC) within the cerebral basal arteries [50], commonly the middle cerebral artery (MCA). Current clinical and research applications include: identifying the MCA and basilar

\footnotetext{
(See figure on next page.)

Fig. 1 Optical coherence tomography (OCT) and optical coherence tomography angiography (OCTA) of the retina. a En face fundus image showing the optic disc and the macula. $\mathbf{b}$ OCT image showing the retinal layers in cross-section passing through the fovea centralis at the location indicated by the bold central arrow in (a). The vitreous (inside of the eye) is at the top of the image and the choroid capillary network (choriocapillaris) is at the bottom. The retinal nerve fibre layer (RNFL) is outlined in red, the inner nuclear layer in blue and the photoreceptor nuclei in yellow, using the manufacturer's segmentation algorithm. c En face OCTA image of the superficial vascular plexus (SVP) at the level of the retinal ganglion cell nuclei (retinal level indicated by the tip of the connecting arrow). $\mathbf{d}$ En face OCTA image of the intermediate capillary plexus (ICP) at the inner border of the inner nuclear layer (retinal level indicated by the connecting arrow tip). e En face OCTA image of the deep capillary plexus (DCP) at the outer border of the inner nuclear layer (retinal level indicated by the connecting arrow tip). $f$ En face OCTA image of the choriocapillaris (retinal level indicated by the connecting arrow tip)
} 


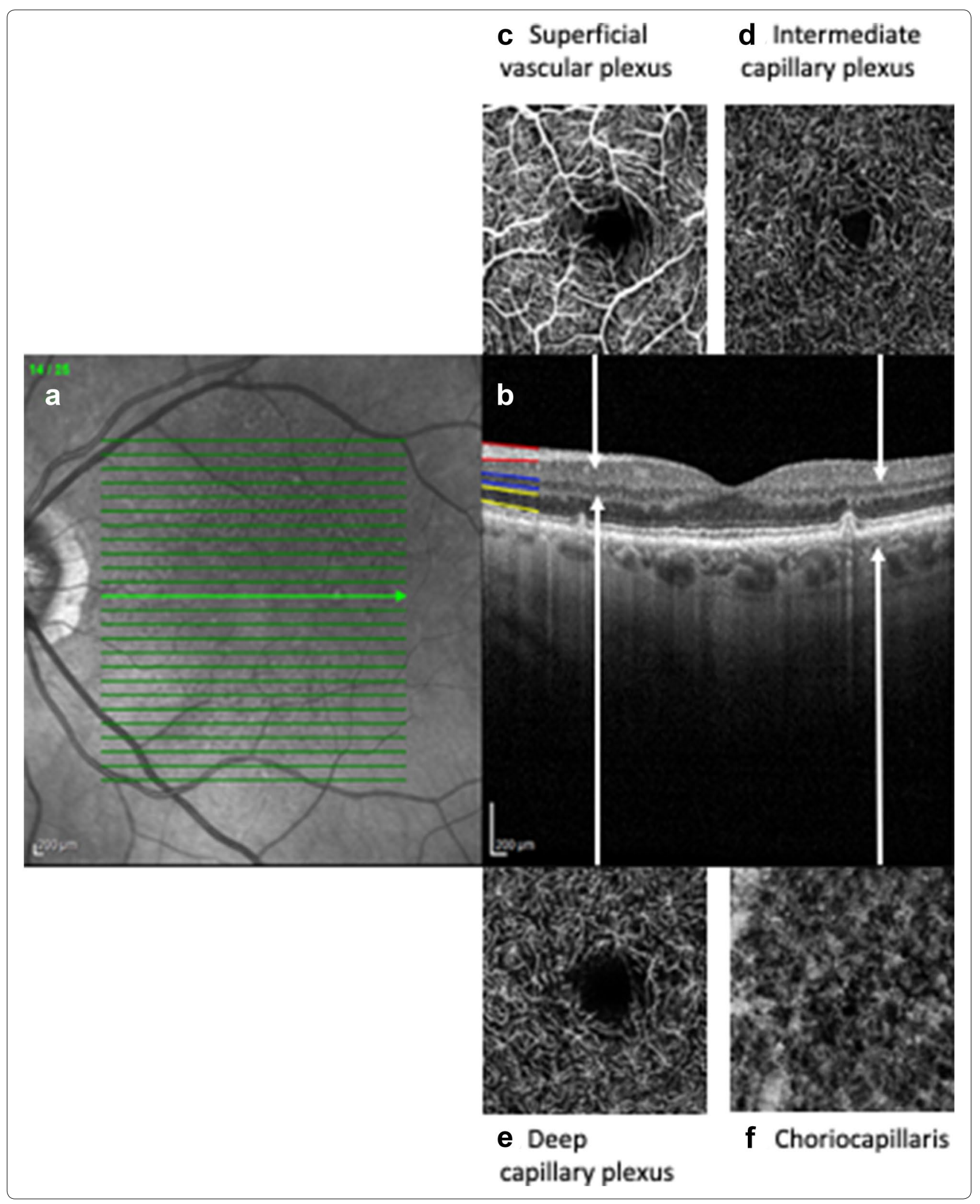


artery vasospasm after subarachnoid haemorrhage, blood flow assessment in the MCA after acute ischaemic stroke, intraoperative monitoring during coronary artery bypass graft, detecting evolving hypoperfusion after traumatic brain injury (TBI), and identifying lower cerebral blood flow velocity in Alzheimer's disease (AD) [50, 51]. TDU demonstrates altered cerebral autoregulation in $50 \%$ of the patients with sepsis and its early stage loss was associated with SABD $[12,52,53]$.

Sidestream dark field (SDF) microscopy provides dynamic bed-side images of surface microcirculation. Illumination is achieved by surrounding a central light guide by concentrically placed light-emitting diodes, providing SDF illumination [54]. Light from the illuminating outer core of the SDF probe penetrates the tissue and illuminates the microcirculation by scattering [54]. SDF requires surface exposure to assess CBF, thus limiting clinical application, but an ovine model of septic shock [10] showed that the onset of septic shock was associated with decreases in cortical cerebral perfused microcirculatory vessel density, the proportion of small perfused vessels and functional capillary density, evidencing reduced microcirculatory flow. These changes were not prevented by fluid administration and were unrelated to changes in MAP and cardiac index, providing evidence of a dissociation between brain perfusion alterations and global perfusion pressure [10]. In a further study, Taccone et al. evaluated the relationship of disturbances in brain tissue oxygenation with microvascular alterations in the ovine septic model [55]. Cerebral functional capillary density and proportion of small perfused vessels significantly decreased from baseline to septic shock onset. Brain lactate:pyruvate ratio (a measure of tissue hypoxia) was increased and brain oxygen tension reduced, likely due to impaired microvascular perfusion [55].

\section{Assessment of the retina and retinal blood flow \\ OCT}

Optical coherence tomography (OCT) allows non-contact, high-resolution cross-sectional retinal imaging [56]. A low coherence light beam is directed toward the target tissue and split into two paths [57]. While one of the light paths travels to the sample tissue-being scattered and reflected back as it passes through-the other travels to a reference mirror and is also reflected back from a known distance [58]. The two reflected light beams interact to produce interference patterns-which depend on the different path lengths-and amplitude information, which makes up the axial scan (A-scan) [59]. Multiple adjacent A-scans captured at several depths combine to produce a 2-dimensional B-scan. Adjacent B-scans form a volumetric retinal image.

Time-domain OCT (TD-OCT) was the first developed OCT which required a moving reference mirror, so had a scan rate of only 400 A-scans per second and a resolution of $8-10 \mu \mathrm{m}$ [56].

Spectral-domain OCT (SD-OCT, a type of Fourier domain) followed, managing 20,000-130,000 A-scans per second and a resolution of $5-7 \mu \mathrm{m}$, by detecting multiple frequencies of light simultaneously (Optopol REVO NX OCT/OCTA, Spectrum, UK) [56].

Swept-source OCT (SS-OCT, also Fourier domain) uses a tunable laser light source, varying the emitted frequency to derive reflectivity data for each wavelength [60]. This increases signal quality in deep tissue compared to SD techniques, because of the greater penetrance of longer wavelengths.

\section{Laser Doppler velocimetry}

The first study attempting to quantify retinal blood flow in humans in 1985 used bidirectional laser Doppler velocimetry (LDV) [61] to measure retinal blood flow velocity and vessel diameters from fundus images, with arteriolar diameters at the site of LDV measuring between 39 and $134 \mu \mathrm{m}$ and venules measuring from 64-177 $\mu \mathrm{m}$. However, this and subsequent studies show high variability in mean blood flow, which is most likely explained by inter-individual variability and the fact that LDV requires good fixation by the participant for up to $45 \mathrm{~min}$ [61-63]. LDV would, therefore, be unsuitable for use in most clinical settings.

Doppler OCT (DOCT) gives quantitative volumetric information on blood flow in arteries and veins [64], but not the retinal microcirculation. However, there are often errors in vessel diameter extraction due to shadowing effects behind the vessel obscuring the boundary [65]. Further, eye movement alters the Doppler angle, causing artefact and limiting clinical application to date [65].

\section{Fundus photography and fundus fluorescein angiography}

Fundus photography is used extensively in ophthalmology, with retinal fundus colour imaging allowing retinal vascular evaluation [66], and is now possible using smartphone attachments which allows portability [67]. However, classifying arteries and veins relies on the colour and diameter of the blood vessels, which may be unreliable between images and does not directly assess microcirculation [66].

Fundus fluorescein angiography (FFA) has been used to image retinal blood flow after intravenous fluorescein injection since the 1930s [68], and images the superficial retinal vasculature, which can be obscured by leakage or haemorrhage from surrounding capillaries [69] and which itself obscures the deeper vasculature [70]. It is therefore not routinely possible to image all retinal capillary layers using FFA [70]. 


\section{OCT angiography}

OCT angiography (OCTA), developed from OCT, uses moving $\mathrm{RBC}$ as an intrinsic contrast medium to give 3-dimensional images of retinal and choroidal blood flow [71] without the need for injectable contrast [72]. OCTA is non-contact, non-invasive, faster and cheaper to run than FFA, with no risk of morbidity from allergic reactions to fluorescein [73], although it does not provide direct information on vascular permeability. Unlike FFA, OCTA is the result of mathematic algorithms which allow estimation of reflectivity and ultimately, for OCTA, allow blood flow detection in arteries, veins and capillaries $[74,75]$. Algorithms utilise the component differences of the varying B-scans [76]. For instance, the OCT signals of SD-OCT and SS-OCT contain intensity (the strength of reflected signal) and phase (the time taken for the reflected signal to return) information; therefore algorithms may be based on intensity, phase, or both intensity and phase of OCT signals, to determine blood flow [77].

Examples of other approaches used for OCTA include: split-spectrum amplitude decorrelation angiography (analyses amplitude changes of the OCT signal, while splitting the spectrum reduces bulk-motion noise [78]); optical microangiography (includes directional information); and OCTA ratio analysis (intensity ratio calculation improves microvasculature detection sensitivity $[77,77])$.

OCTA is now used alongside OCT and FFA in the diagnosis and management of numerous retinal diseases [80], including age-related macular degeneration and diabetic retinopathy [81], and in animal research [82]. Recent developments in OCT and OCTA increase portability and show feasibility for use in a critical care setting and therefore the potential to assess retinal blood flow in this group of patients [83], although the number of images may be limited within the context of usual ITU care, and by unconscious patients and semi-conscious patients who may be uncooperative and prevent imaging entirely [83]. In an ITU clinical environment, two operators are needed to acquire the scans, the devices are bulky, and given the significant cost of the device, it needs to be clearer that it provides significant value in terms of its performance, feasibility and utility in the ITU environment, including on ventilated patients.

OCTA is not without its limitations, probably the most significant being scan artefacts caused by eye movement, or projection artefacts from other retinal vessels. Further, artefacts show up differently on the scan depending on what caused them, so it is important to be able to distinguish between them. Motion artefacts from blinking show up as dark lines, while artefacts from eye movements show up as horizontal white lines [84]. These can be reduced with use of an incorporated eye-tracker, although may still increase acquisition time [84].
Projection artefacts can result when superficial blood vessels obscure deeper layers, leading to inaccurate interpretation of deeper vessel blood flow. OCTA platforms have endeavoured to reduce this by incorporating projection-masking software, but are unable to minimise projections in all layers [84].

The many different algorithms used to detect blood flow and segment retinal layers and capillary boundaries [77] make comparison of OCTA studies between devices difficult [74]. Retinal layer segmentation can also be inaccurate, which may be apparent as dark areas on the en face OCTA image, requiring manual adjustment prior to final interpretation [84]. It is also possible for flow to be incorrectly detected using OCTA, relating to the time difference between successive B-scans. Normal SDOCTA has an interscan time of only $5 \mathrm{~ms}$, so if the flow is too slow or fast then the B-scans would display no difference, and therefore show no flow [85].

While the image produced by an OCTA scan shows the presence or absence of blood flow, it does not give information on the speed, direction, or volume. Most commercial devices do not include automated calculation of these characteristics and the measurements are not uniform across devices which do [85], creating difficulty when comparing studies. It is therefore necessary for some studies to use third party software to quantify the data, such as measuring the FAZ area and perimeter [85], or by using either the binary or skeletonised images to calculate: perfusion density; vessel length density; and fractal dimension [86].

Finally, as OCTA is relatively new, normative data are developing [87-89], with some unknowns regarding the correlation between general parameters and vessel density [90]. There is systematic variation in FAZ area between devices (measuring higher with Heidelberg than Canon devices) but with a very high intraclass correlation coefficient (ICC) of 0.96 [91], compared to an ICC for flow index of 0.62-0.67 [92] and vessel density of $0.74-$ 0.81 [93].

\section{Association of retinal and cerebral neurodegeneration}

Structural retinal imaging techniques demonstrate retinal changes associated with systemic disease (Table 1) [14]. Cerebral neurodegeneration is associated with retinal neurodegeneration in acute and chronic insults, including stroke (Merge EyeScanner camera) [94], Parkinson's disease (PD; RTVue XR Avanti SD-OCTA) [95], AD (Spectralis OCT and dynamic vessel analyser) [96, 97] and Huntington's disease (HD; Heidelberg Spectralis OCT) [98].

With cerebral vasculature implicated in various neurodegenerative disorders, retinal neurodegeneration and vasculature manifestations of these disorders inform the 


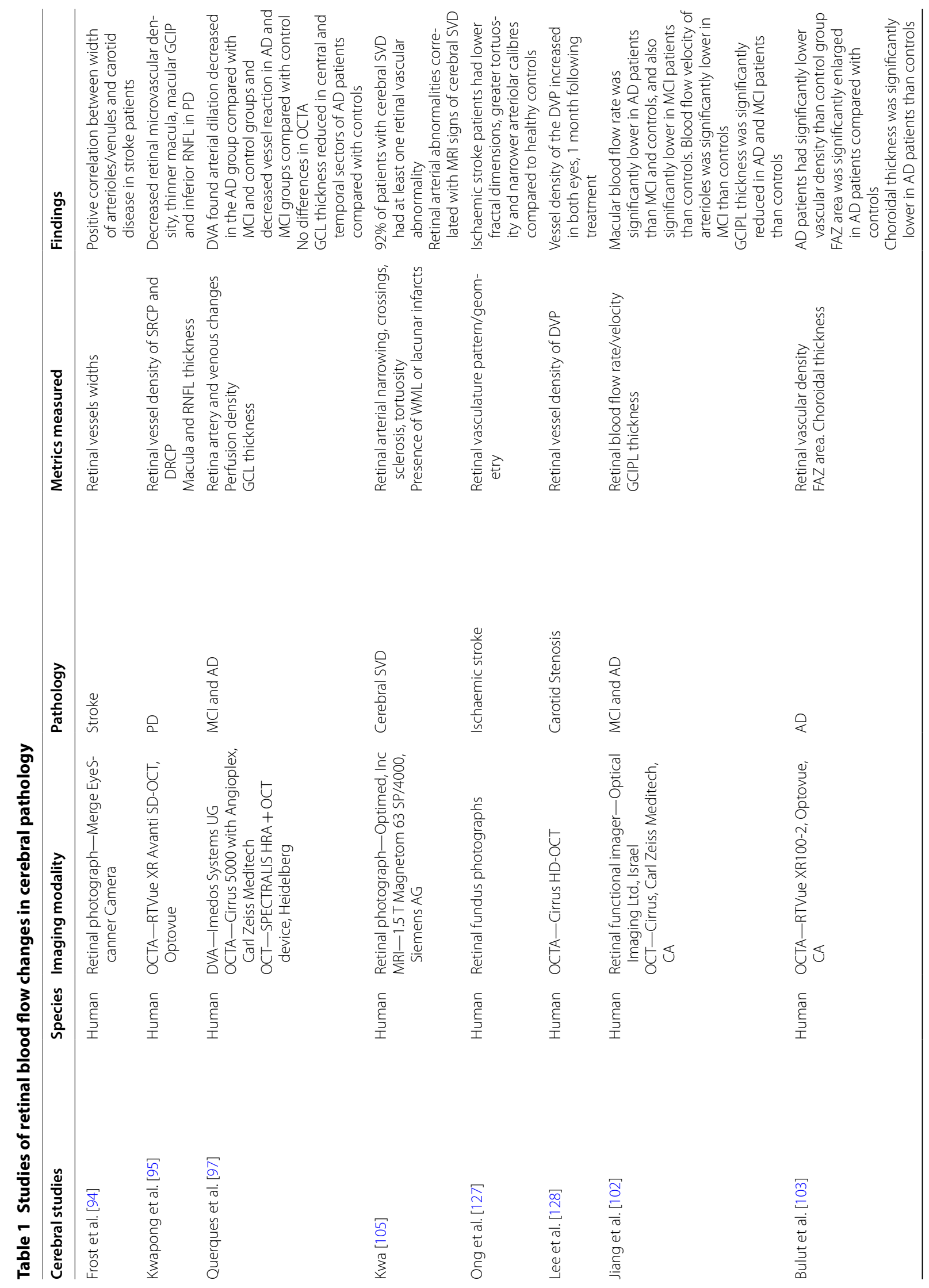




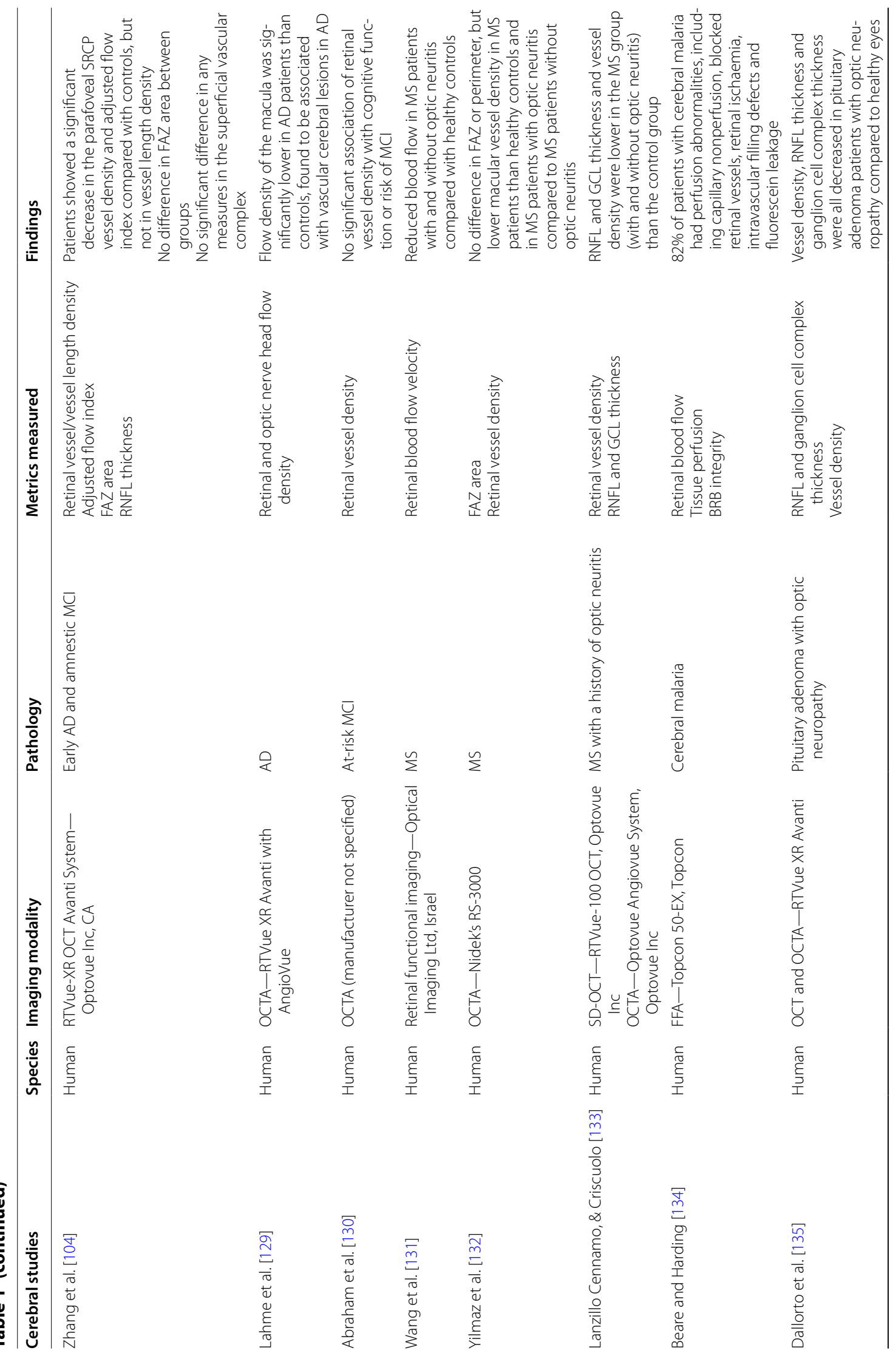




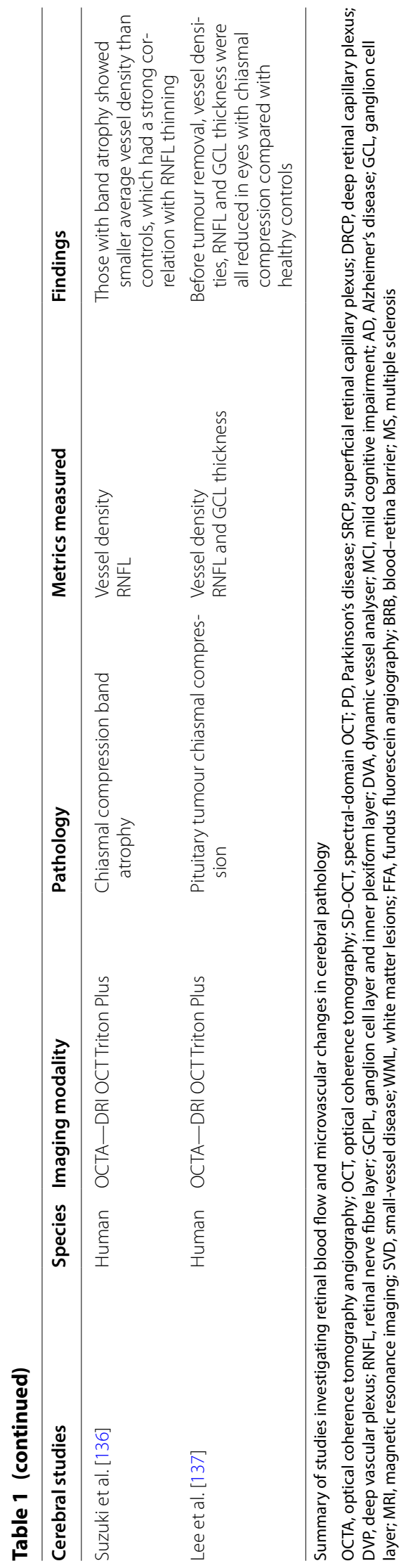


retinal-cerebral blood flow relationship [99]. In PD, there was reduced retinal microvascular density in the superficial capillary layer of PD patients compared to healthy controls, suggesting either that PD may associate with cerebral small-vessel disease (SVD), as seen in autopsy studies, or that PD-associated retinal neurodegeneration reduces retinal blood flow [95]. In PICS associated with cognitive impairment [100], the retinal vascular changes during acute illness and afterwards may similarly mirror cerebral hypoperfusion and microvascular dysfunction.

OCT demonstrates retinal structural changes in AD patients compared with healthy individuals, including GCL loss [101] and ganglion cell inner plexiform layer loss in certain sections of the retina [102]. In patients with mild cognitive impairment (early AD) and established AD, OCTA showed lower retinal blood flow by measuring blood flow rate and blood flow velocity in both retinal arteries and veins, showing lower vascular density in the macular, foveal and parafoveal zones and larger FAZ areas compared to cognitively normal patients [102-104].

In a study investigating the relationship between retinal arterial disease and cerebral SVD, 60\% of patients with a systemic atherosclerotic disease showed signs of cerebral SVD on MRI [105]. 92\% of these individuals had at least one retinal arterial abnormality irrespective of the presence of hypertension, suggesting that retinal signs are more sensitive than SVD on cerebral MRI in detecting cerebrovascular disease [105].

Cerebral neurodegenerative disorders cause retinal structural changes and secondary retinal blood flow changes, whilst cerebrovascular disease also reduces retinal perfusion, providing evidence that pathological changes to cerebral perfusion and cerebral neurodegeneration both affect retinal perfusion. This is particularly relevant to critical illness in which cerebral hypoperfusion or hyperperfusion may be both caused by and contribute to cerebral dysfunction and damage, and altered retinal blood flow may relate to both systemic hypoperfusion and sepsis-induced neurodegeneration [22].

\section{Conjunctival and sublingual microcirculation in sepsis}

Techniques to monitor surface microcirculatory changes directly include: SDF videomicroscopy which developed from orthogonal polarisation spectral (OPS); incident dark field (IDF) imaging; laser Doppler perfusion imaging (LDPI); and laser speckle contrast imaging (LSCI) [106]. OPS demonstrates reduced sublingual microvascular blood flow in patients with severe sepsis by direct visualisation, and correlated microvascular alterations with survival of septic patients [107], while SDF demonstrates hypoperfusion and increased heterogeneity in septic microcirculation [108]. The sublingual area is the site used most to evaluate microcirculation in critically ill patients, with SDF the current standard method to do this [109]. With the introduction of handheld video microscopes, SDF also allows bedside monitoring of microcirculation, but it is not yet widely used in clinical practice [8]. A major drawback of SDF is that it can only monitor skin and mucosal blood flow and requires direct contact with the skin, causing pressure and motion artefacts, posing technical challenges which reduces video quality and reliability [110].

IDF uses a green light source that is absorbed by haemoglobin to detect RBC [34] with devices optimised for surface microcirculatory visualisation and may have better image quality than SDF imaging [111]. Portable IDF (Cytocam ${ }^{\circledR}$-IDF device) demonstrated reductions in all microcirculatory parameters of the conjunctiva, including microvascular flow index (MFI) and total and perfused vessel density, in septic patients compared with healthy individuals [34]. Similarly, in the ovine septic and haemorrhagic shock model, functional capillary density and MFI of the conjunctiva capillary microcirculation were significantly reduced in septic shock, with alterations correlating with sublingual capillary microcirculation [112]. SDF in a pig sepsis model (Microscan; Microvision Medical) showed significant decreases in MFI and proportion of perfused small vessels (venules and capillaries with diameters $<20 \mu \mathrm{m}$ ) in the conjunctival, sublingual, jejunal and rectal mucosal microcirculation following sepsis onset [113].

LDPI and LSCI are non-contact techniques, but measure average Doppler shift and therefore only assess relative flow changes, normalised to baseline values [106]. In contrast, OCTA requires no contact and has recently shown suitability for evaluating sublingual microcirculation in healthy volunteers, suggesting it is a promising method for peripheral (as well as retinal) microcirculatory evaluation [8].

\section{Retinal blood flow changes associated with systemic pathology Retinal blood flow in cardiovascular and inflammatory disease}

Retinal changes in malignant hypertension are well-recognised. When patients with malignant hypertension were compared to controls, some, but not all, measures of vessel density and skeletal density of the superficial retinal layer and deep retinal layer were reduced in the hypertensive group, demonstrating retinal capillary dropout associated with malignant hypertension using OCTA [114]. Retinal capillary density was reduced in the DVP of patients with poorly controlled blood pressure compared with those with well controlled blood pressure, further highlighting the potential role of OCTA to monitor early 
microvascular changes arising from systemic hypertension [15]. Further studies would answer the extent of which these changes are associated with microvascular complications and end-organ damage [114].

Patients with atrial fibrillation have abnormal retinal electrophysiological responses and lower flow density in the macular and ON SVP on OCTA compared with healthy controls, which partially normalised when patients were restored to sinus rhythm, but showed no evidence of a difference in FAZ area [115].

Patients suffering acute coronary syndromes also have abnormal retinal blood flow on OCTA, with the lowest inner retinal vessel density in the highest risk patients (highest American Heart Association scores and the lowest left ventricular ejection fractions) [116]. Some earlystage coronary heart disease patients could be defined as a high-risk population on OCTA by reduced retinal vessel density, and reduced choroidal vessel density and blood flow, suggesting an efficient and non-invasive method for detection of early-stage coronary heart disease [117]. Taken together, the findings in cardiac disease suggest that impaired cardiac output reduces retinal blood flow, especially given the partial normalisation when sinus rhythm is restored. However, the previous studies demonstrating preserved, autoregulated retinal blood flow under hypovolaemic stress also suggest that, common to the studies of systemic and cerebrovascular disease, at least some of the OCTA abnormalities observed reflect a long-term vasculopathy.

There was no association between a diagnosis of Crohn's disease or ulcerative colitis and retinal blood flow, but when either group of patients had active disease, FAZ area was reduced compared to patients in remission, suggesting altered retinal blood flow autoregulation by systemic inflammatory status [16]. Systemic sclerosis has involvement of the microvasculature as one of the earliest features. OCTA showed significantly decreased foveal, parafoveal and perifoveal vessel densities in the superficial capillary plexus, and foveal vessel density in the DCP, of patients with systemic sclerosis compared with healthy individuals [118]. These results suggest indicators of retinal vascular injury before patients become symptomatic [118].

Pregnancy is a state with hyperdynamic circulation and a finely modulated immune system [119]. Pre-eclampsia is associated with generalised endothelial dysfunction, increasing vascular resistance and leakage from blood vessels and manifesting as hypertension, proteinuria and oedema, but no microcirculatory changes detectable by SDF [17]. In contrast, patients in the third trimester of pregnancy have reduced macular SVP vessel density. Macular SVP and ICP vessel density in high-risk pregnancies are also lower than in low-risk pregnancies, and patients with pre-eclampsia also have reduced macular SVP and ICP, but increased peripapillary SVP perfusion compared to patients with uncomplicated pregnancy and normal controls [120].

A prototype handheld SS-OCTA device was used to capture high-quality vitreoretinal images in awake premature neonates at risk for retinopathy of prematurity, with greater imaging speed and detail compared with currently available handheld SD-OCT devices [121].

\section{Retinal microcirculation in sepsis and haemorrhagic shock}

In a pig model of acute respiratory distress syndrome [122], RNFL thickness was increased and there was immunostaining for reactive oxygen species HIF- $1 \alpha$ and VEGF-A in retinal arterioles, suggestive of increased retinal vascular permeability and endothelial dysfunction [122].

After ovine haemorrhagic shock [123], SVP flow density on OCTA decreased from $44.7 \%$ baseline to $34.5 \%$, recovering to $46.9 \%$ after fluid resuscitation, correlating with systemic haemodynamic parameters. Conjunctival microcirculation assessed using IDF microscopy also showed a reduced proportion of perfused vessels from $100 \%$ to $72 \%$, which returned to $98.7 \%$ after resuscitation [123]. The alterations in OCTA flow density correlated with reduced perfused vessel density in IDF of the conjunctiva and haemodynamic parameters (MAP, heart rate and cardiac index all decreased), suggesting that both the retinal and conjunctival microcirculatory changes may relate to cerebral perfusion alterations. In contrast, in a rat haemorrhagic shock model, choroidal blood flow dropped in proportion to MAP (preceding increases in serum lactate), but retinal blood flow assessed by OCTA was maintained [124].

FFA in patients with sepsis demonstrated prolonged retinal arterial filling time after intravenous dye injection, associated with fundus signs of retinal vasculopathy including haemorrhages and microaneurysms, although retinal arteriolar diameters were not measured [125]. Patients with delayed retinal arterial filling had a lower cardiac index, higher Acute Physiology and Chronic Health Evaluation II scores and lower interleukin- 6 and C-reactive protein levels, suggesting an impaired inflammatory response [125].

Septic patients in the ITU had increased average retinal arteriolar calibres $(165 \mu \mathrm{m}[149-187 \mu \mathrm{m}]$ vs. $146 \mu \mathrm{m}[142-158 \mu \mathrm{m}], p=0.002)$ compared with healthy controls and decreased vascular length density $(0.51 \%$ vs. $0.64 \%, p<0.001)$ on portable fundus photography compared with healthy controls [126].

There is a need for improved monitoring of cerebral perfusion in a critical care environment to allow perfusion-directed resuscitation, improve patient outcomes, 


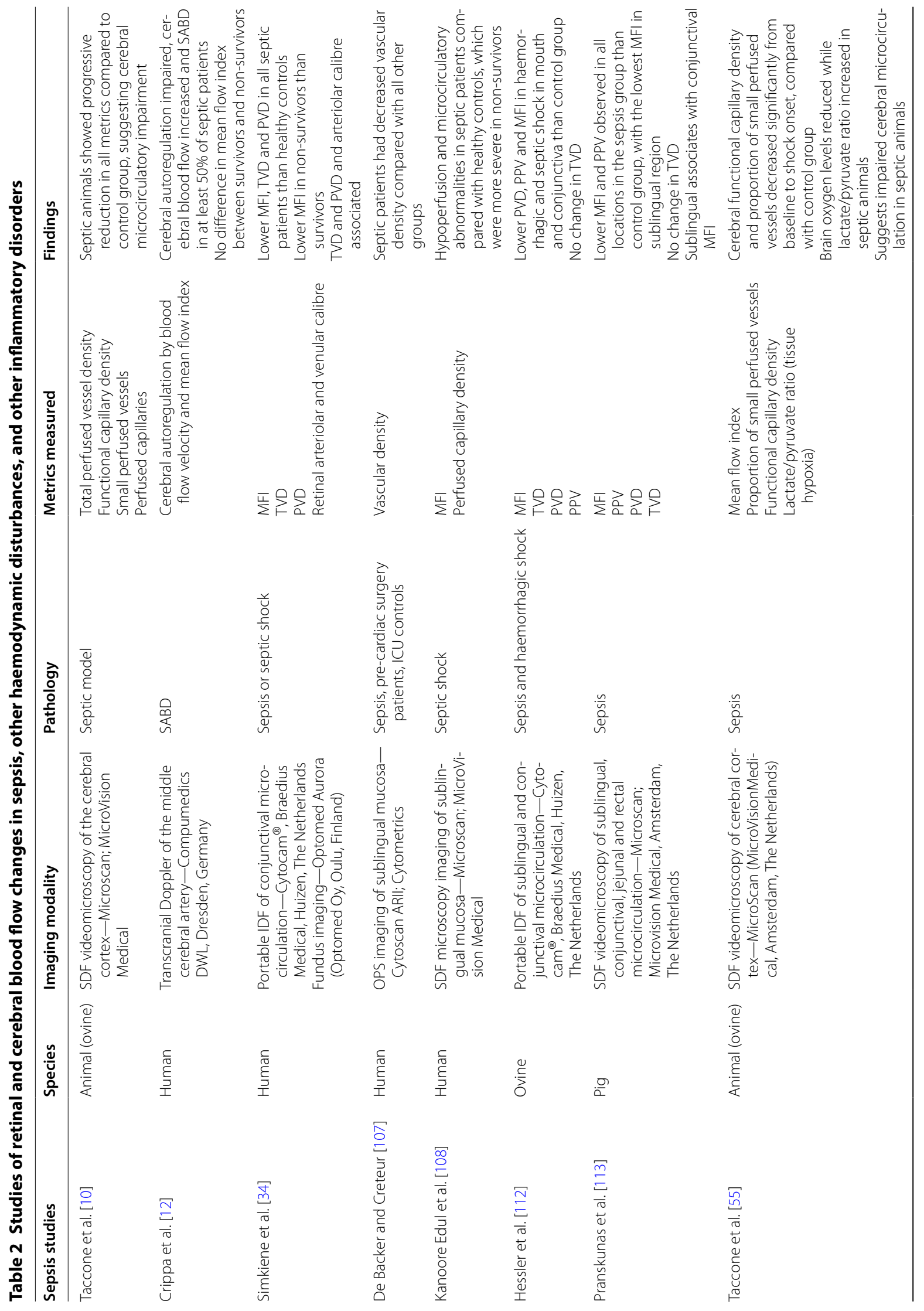




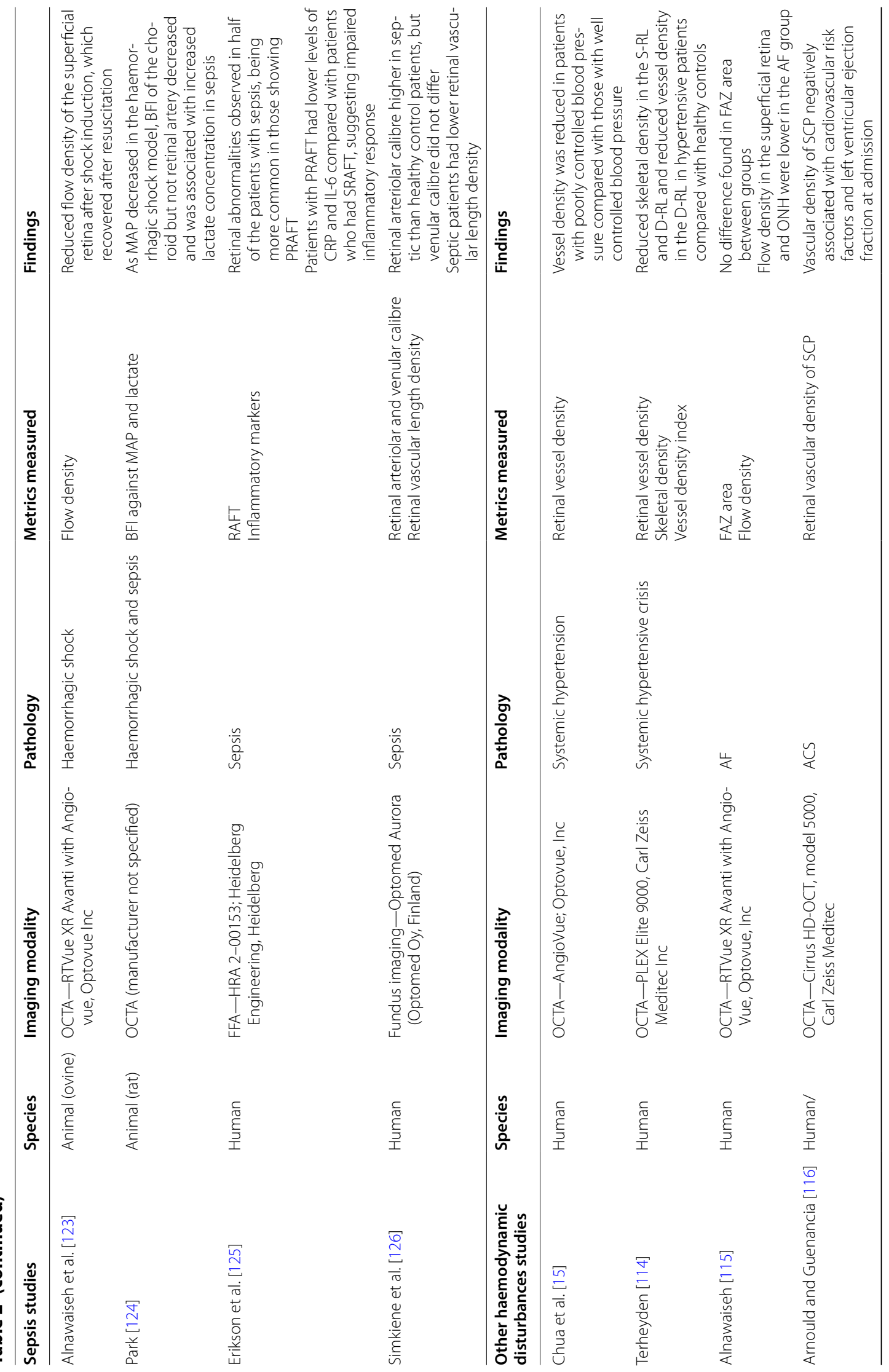




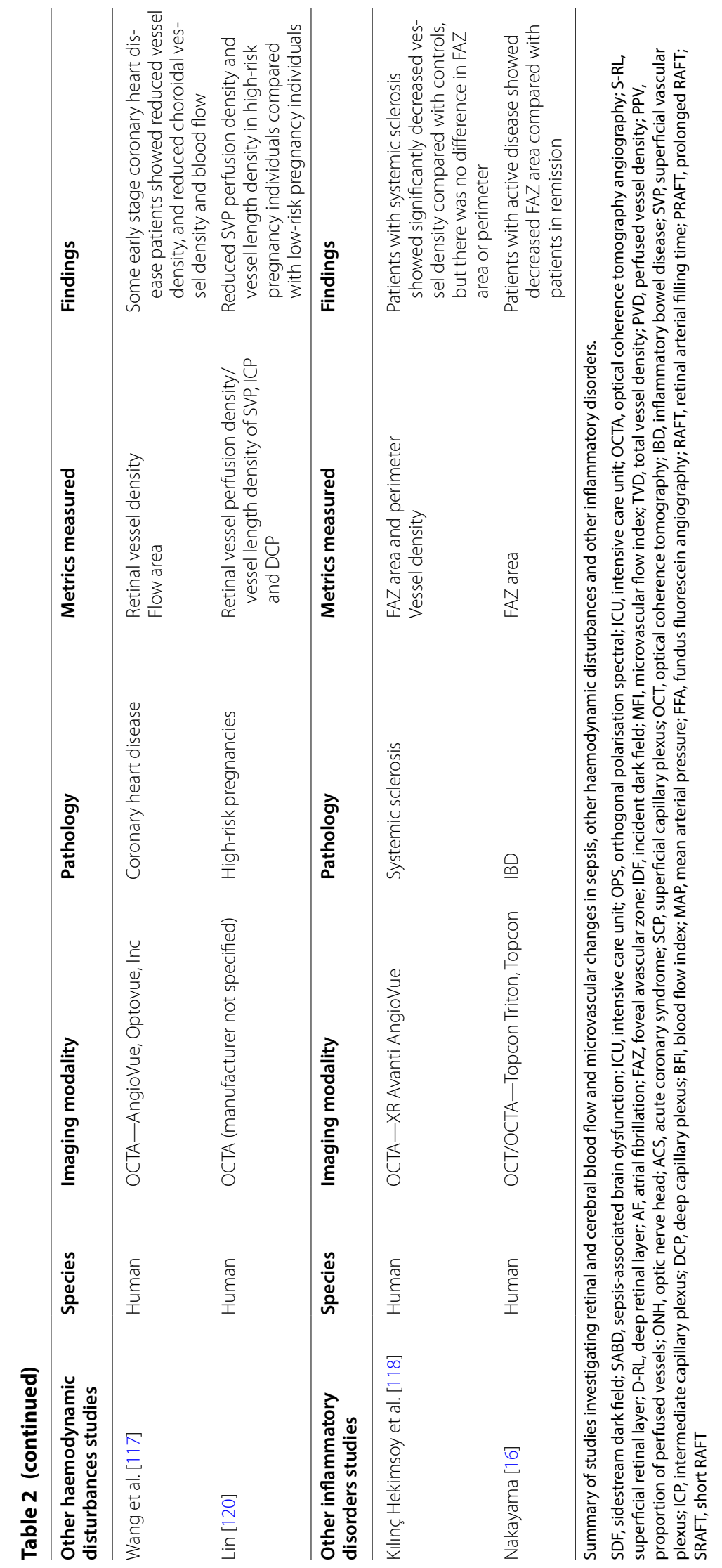


and possibly reduce long-term cognitive impairment. These retinal imaging studies demonstrate that retinal vessel density and retinal perfusion are affected by systemic haemodynamic changes [116], and the systemic inflammatory response $[16,118]$, but also that it does not simply provide a mirror to systemic haemodynamic status, being resistant to change in some models [124] and providing additional information in others (Table 2) [117, 120, 125].

\section{Conclusions}

The reviewed studies demonstrate the link between retinal and cerebral blood flow, and that changes in retinal perfusion reflect changes in cerebral microcirculation. Retinal blood flow is altered by systemic and microcirculatory hypoperfusion, and is in association with cerebral and retinal neurodegeneration. Conjunctival and sublingual microcirculation are also altered in sepsis. Of the different retinal blood flow imaging modalities, OCTA is the least invasive and is a promising method for retinal evaluation in the future. Retinal blood flow, therefore, has potential as a biomarker of systemic disease, with developing evidence in critical illness and sepsis.

\begin{abstract}
Abbreviations
ITU: Intensive treatment unit; PICS: Post-intensive care syndrome; MAP: Mean arterial pressure; SABD: Sepsis-associated brain dysfunction; CBF: Cerebral blood flow; CPP: Cerebral perfusion pressure; ICP: Intracranial pressure; BBB: Blood-brain barrier; EC: Endothelial cells; NO: Nitric oxide; nNOS: Neuronal nitric oxide synthase; RPCP: Radial peripapillary capillary plexus; SVP: Superficial vascular plexus; GCL: Ganglion cell layer; ICP: Intermediate capillary plexus; DCP: Deep capillary plexus; SVC: Superficial vascular complex; DVC: Deep vascular complex; FAZ: Foveal avascular zone; RNFL: Retinal nerve fibre layer; ON: Optic nerve; IOP: Intraocular pressure; BRB: Blood-retina barrier; CTP: Computed tomography perfusion; ${ }^{15} \mathrm{O}$ : Triple oxygen; ${ }^{15} \mathrm{OPET}$ : Triple oxygen positron emission tomography; SPECT: Single-photon emission computed tomography; MRA: Magnetic resonance angiography; NIRS: Near-infrared spectroscopy; fMRI: Functional magnetic resonance imaging; TDU: Transcranial Doppler ultrasound; RBC: Red blood cells; MCA: Middle cerebral artery; TBI: Traumatic brain injury; AD: Alzheimer's disease; OCT: Optical coherence tomography; A-scan: Axial scan; TD-OCT:Time-domain optical coherence tomography; SD-OCT: Spectral-domain optical coherence tomography; SS-OCT: Swept-source optical coherence tomography; LDV: Laser doppler velocimetry; DOCT: Doppler optical coherence tomography; FFA: Fundus fluorescein angiography; OCTA: Optical coherence tomography angiography; ICC: Intraclass correlation coefficient; PD: Parkinson's disease; HD: Huntington's disease; SVD: Small vessel disease; SDF: Sidestream dark field; OPS: Orthogonal polarisation spectral; IDF: Incident dark field; LDPI: Laser Doppler perfusion imaging; LSCl.
\end{abstract}

\section{Acknowledgements}

This study/project is funded by the National Institute for Health Research (NIHR) Surgical Reconstruction and Microbiology Research Centre (SRMRC). The views expressed are those of the author(s) and not necessarily those of the NIHR or the Department of Health and Social Care.

\section{Authors' contributions}

EC was a major contributor in writing the manuscript. All authors read and approved the final manuscript.

\section{Funding}

This project is funded by the National Institute for Health Research (NIHR) Surgical Reconstruction and Microbiology Research Centre (SRMRC). The views expressed are those of the authors and not necessarily those of the NIHR or the Department of Health and Social Care.

\section{Availability of data and materials}

Not applicable.

Ethics approval and consent to participate

Not applicable.

\section{Consent for publication}

Not applicable.

\section{Competing interests}

The authors declare that they have no competing interests.

\section{Author details}

${ }^{1}$ Neuroscience and Ophthalmology, Institute of Inflammation and Ageing, College of Medical and Dental Sciences, University of Birmingham, Birmingham, UK. ${ }^{2}$ Ophthalmology Department, University Hospitals Birmingham NHS Foundation Trust, Birmingham, UK. ${ }^{3}$ NIHR Surgical Reconstruction and Microbiology Research Centre, University Hospitals Birmingham NHS Foundation Trust, Birmingham, UK. ${ }^{4}$ Critical Care Unit, University Hospitals Birmingham NHS Foundation Trust, Birmingham, UK. ${ }^{5}$ Birmingham Acute Care Research Group, Institute of Inflammation and Ageing, College of Medical and Dental Sciences, University of Birmingham, Birmingham, UK. ${ }^{6}$ Axolotl Consulting Ltd, Droitwich WR9 0JS, Worcestershire, UK. ${ }^{7}$ Division of Biomedical Sciences, Warwick Medical School, University of Warwick, Coventry CV4 7HL, UK. ${ }^{8} \mathrm{NIHR}$ Biomedical Research Centre for Ophthalmology, Moorfields Eye Hospital NHS Foundation Trust and UCL Institute of Ophthalmology, London, UK. ${ }^{9}$ Centre for Rare Diseases, Institute of Translational Medicine, Birmingham Health Partners, Birmingham, UK. ${ }^{10}$ Academic Department of Military Surgery and Trauma, Royal Centre for Defence Medicine, Birmingham, UK.

Received: 27 May 2020 Accepted: 23 October 2020

Published online: 12 November 2020

\section{References}

1. Bäckman CG, Ahlberg M. Group meetings after critical illness-giving and receiving strength. Intensive Crit Care Nurs. 2018;46:86-91.

2. Angus DC, van der Poll T. Severe sepsis and septic shock. N Engl J Med. 2013;369:840-51.

3. Rudd K, Johnson S, Agesa A, et al. Global, regional, and national sepsis incidence and mortality, 1990-2017: analysis for the Global Burden of Disease Study. Lancet. 2020;395:200-11.

4. De Backer D, Orbegozo Cortes D. Pathophysiology of microcirculatory dysfunction and the pathogenesis of septic shock. Virulence. 2014:5:73-9.

5. Trzeciak S, McCoy JV, Phillip Dellinger R, et al. Early increases in microcirculatory perfusion during protocol-directed resuscitation are associated with reduced multi-organ failure at $24 \mathrm{~h}$ in patients with sepsis. Intensive Care Med. 2008;34:2210-7.

6. Rawal G, Yadav S, Kumar R. Post-intensive care syndrome: an overview. J Transl Intern Med. 2017;5:90-2.

7. Asfar P, Meziani F, Hamel J-F, et al. High versus low blood-pressure target in patients with septic shock. N Engl J Med. 2014;370:1583-93.

8. Hessler M, Nelis P, Ertmer C, et al. Optical coherence tomography angiography as a novel approach to contactless evaluation of sublingual microcirculation: a proof of principle study. Sci Rep Nat Res. 2020;10:5408

9. De Backer D, Donadello K, Sakr Y, et al. Microcirculatory alterations in patients with severe sepsis: impact of time of assessment and relationship with outcome. Crit Care Med. 2013;41:791-9.

10. Taccone FS, Su F, Pierrakos C, et al. Cerebral microcirculation is impaired during sepsis: an experimental study. Crit Care. 2010;14:R140. 
11. Burkhart CS, Siegemund M, Steiner LA. Cerebral perfusion in sepsis. Crit Care. 2010;14:215.

12. Crippa IA, Subirà C, Vincent JL, et al. Impaired cerebral autoregulation is associated with brain dysfunction in patients with sepsis. Crit Care. 2018;22:327.

13. Donnelly J, Budohoski KP, Smielewski P, et al.: Regulation of the cerebral circulation: bedside assessment and clinical implications. Crit Care 2016; 18

14. Schwartz $M$, London A. The retina as a window to the brain-from eye research to CNS disorders. Nat Rev Neurol. 2013;9:44-53.

15. Chua J, Chin CWL, Hong J, et al. Impact of hypertension on retinal capillary microvasculature using optical coherence tomographic angiography. J Hypertens. 2019;37:572-80.

16. Nakayama L. Retinal Avascular Foveal Zone as a systemic biomarker to evaluate inflammatory bowel disease control. Invest Ophthalmol Vis Sci. 2019;60:4572.

17. Cornette J. Microcirculation in women with severe pre-eclampsia and HELLP syndrome: a case-control study. BJOG. 2014;121:363-70.

18. Wagner SK, Fu DJ, Faes L, Liu X, Huemer J, Khalid H, Ferraz D, Korot E, Kelly C, Balaskas K, Denniston AK, Keane PA. Insights into systemic disease through retinal imaging-based oculomics. Trans Vis Sci Tech. 2020;9(2):6.

19. Fantini S. Cerebral blood flow and autoregulation: current measurement techniques and prospects for noninvasive optical methods. Neurophotonics. 2016;3:1-31.

20. Cipolla MJ. The cerebral circulation. Morgan and claypool life sciences. California: Morgan and Claypool Life Sciences; 2010.

21. Peterson EC, Wang Z, Britz G. Regulation of cerebral blood flow. Int J Vasc Med. 2011;2011:823525.

22. Goodson CM, Rosenblatt K. Cerebral blood flow autoregulation in sepsis for the intensivist: why its monitoring may be the future of individualized care. J Intensive Care Med. 2018;33:63-73.

23. Peyrounette $M$, Davit Y. Multiscale modelling of blood flow in cerebral microcirculation: details at capillary scale control accuracy at the level of the cortex. PLoS ONE. 2018;13:e0189474.

24. Kolinko Y, Krakorova K. Microcirculation of the brain: morphological assessment in degenerative diseases and restoration processes. Rev Neurosci. 2014:26:75-93.

25. Muoio V, Persson PB, Sendeski MM. The neurovascular unit—concept review. Acta Physiol. 2014;210:790-8.

26. Winkler EA, Bell RD, Zlokovic BV. Central nervous system pericytes in health and disease. Nat Neurosci. 2011;14:1398-405.

27. Talman WT, Nitschke Dragon D. Neuronal nitric oxide mediates cerebral vasodilatation during acute hypertension. Brain Res. 2007;1139:126-32.

28. Peppiatt CM, Howarth C, Mobbs P, et al. Bidirectional control of CNS capillary diameter by pericytes. Nature. 2006:443:700-4.

29. Rosengarten B, Wolff S. Effects of inducible nitric oxide synthase inhibition or norepinephrine on the neurovascular coupling in an endotoxic rat shock model. Crit Care. 2009;13:R139.

30. Campbell JP, Zhang M, Hwang TS, et al. Detailed vascular anatomy of the human retina by projection-resolved optical coherence tomography angiography. Sci Rep. 2017;7:42201.

31. Snell R, Lemp M. Clinical anatomy of the eye. 2 nd ed. Oxford: Blackwell Science, Inc; 1998.

32. Samara WA, Say EAT, Khoo CTL, et al. Correlation of foveal avascular zone size with foveal morphology in normal eyes using optical coherence tomography angiography. Retina. 2015;35:2188-95.

33. Remington LA. Orbital Blood Supply. Clin Anat Physiol Vis Syst. 2011;202-17

34. Simkiene J, Pranskuniene Z, Vitkauskiene A, et al. Ocular microvascular changes in patients with sepsis: a prospective observational study. Ann Intensive Care. 2020;10:38

35. Bill A, Sperber GO. Control of retinal and choroidal blood flow. Eye. 1990;4:319-25.

36. Pournaras CJ. Regulation of retinal blood flow in health and disease. Prog Retin Eye Res. 2008;27:284-330

37. Fenstermacher JD. Cerebral Microcirculation. Encycl Neurol Sci. 2014

38. Harris A, Ciulla TA. Regulation of Retinal and Optic Nerve Blood Flow. Arch Ophthalmol. 1998;116:1491-5.

39. Luo X, Shen YM: Ocular Blood Flow Autoregulation Mechanisms and Methods. J Ophthalmol 2015
40. Patton N, Aslam T, Macgillivray T, et al. Retinal vascular image analysis as a potential screening tool for cerebrovascular disease: a rationale based on homology between cerebral and retinal microvasculatures. J Anat. 2005;206:319-48.

41. Pi S, Hormel TT, Wei X, Cepurna W, et al. Monitoring retinal responses to acute intraocular pressure elevation in rats with visible light optical coherence tomography. Neurophotonics. 2019;6(4):041104.

42. Cabrera DeBuc D. Retinal microvascular network alterations: potential biomarkers of cerebrovascular and neural diseases. Am J Physiol Heart Circ Physiol. 2017;312:H201-12.

43. Coltman G. Magnetic resonance angiography of the cerebrovascular system. Aust Prescr. 2001;24:435-56.

44. Krainik A, Villien $M$, Troprès I, et al. Functional imaging of cerebral perfusion. Diagn Interv Imaging. 2013;94:1259-78.

45. Brady K, Joshi B, Zweifel C, et al. Real-time continuous monitoring of cerebral blood flow autoregulation using near-infrared spectroscopy in patients undergoing cardiopulmonary bypass. Stroke. 2010;41:1951-6.

46. Rostami E, Engquist $\mathrm{H}$, Enblad P. Imaging of cerebral blood flow in patients with severe traumatic brain injury in the neurointensive care. Front Neurol. 2014;5:114.

47. Aliaga M, Forel J-M, De Bourmont S, et al. Diagnostic yield and safety of CT scans in ICU. Intensive Care Med. 2015;41:436-43.

48. Murkin JM, Arango M. Near-infrared spectroscopy as an index of brain and tissue oxygenation. Br J Anaesth. 2009;103:3-13.

49. MacDonald SPJ, Kinnear FB, Arendts G, et al. Near-infrared spectroscopy to predict organ failure and outcome in sepsis: the assessing risk in sepsis using a tissue oxygen saturation (ARISTOS) study. Eur J Emerg Med. 2019;26:174-9.

50. Purkayastha S, Sorond F. Transcranial doppler ultrasound: technique and application. Semin Neurol. 2012;32:411-20.

51. Naqvi J. Transcranial doppler ultrasound: a review of the physical principles and major applications in critical care. Int J Vasc Med. 2013;2013:629378.

52. Berg RMG, Plovsing RR, Bailey DM, et al. Dynamic cerebral autoregulation to induced blood pressure changes in human experimental and clinical sepsis. Clin Physiol Funct Imaging. 2016;36:490-6.

53. Schramm P, Klein KU, Falkenberg L, et al. Impaired cerebrovascular autoregulation in patients with severe sepsis and sepsis-associated delirium. Crit Care. 2012;16:R181.

54. Goedhart PT. Sidestream Dark Field (SDF) imaging: a novel stroboscopic LED ring-based imaging modality for clinical assessment of the microcirculation. Opt Express. 2007:15:15101-14.

55. Taccone FS, Su F, De Deyne C, et al. Sepsis is associated with altered cerebral microcirculation and tissue hypoxia in experimental peritonitis. Crit Care Med. 2014;42:114-22.

56. Adhi M, Duker JS. Optical coherence tomography-current and future applications. Curr Opin Ophthalmol. 2013;24:213-21.

57. Arevalo JF, Krivoy D, Fernandez CF. How Does Optical Coherence Tomography Work? Basic Principles. In: Arevalo JF, eds. Retinal Angiography and Optical Coherence Tomography. New York: Springer New York; 2009. pp. 217-222

58. Hamdan R. Optical coherence tomography: From physical principles to clinical applications. Arch Cardiovasc Dis. 2012;105:529-34.

59. Bhende M, Shetty S. Optical coherence tomography: A guide to interpretation of common macular diseases. Indian J Ophthalmol. 2018;66:20-35

60. Kishi S. Impact of swept source optical coherence tomography on ophthalmology. Taiwan J Ophthalmol. 2016;6:58-68.

61. Riva CE, Grunwald JE: Blood velocity and volumetric flow rate in human retinal vessels. 1985; pp. 1124-1132

62. Garhofer G. Retinal Blood Flow in Healthy Young Subjects. Investig Opthalmol Vis Sci. 2012;53:698-703.

63. Garcia JPS Jr, Garcia PT. Retinal blood flow in the normal human eye using the canon laser blood flowmeter. Ophthalmic Res. 2002;34:295-9.

64. Huang S, Shen M, Zhu D, et al. In vivo imaging of retinal hemodynamics with OCT angiography and Doppler OCT. Biomed Opt Express. 2016;7:663-76.

65. Leitgeb RA, Werkmeister RM, Blatter C, et al. Doppler optical coherence tomography. Prog Retin Eye Res. 2014;41:26-43. 
66. Miri M, Amini Z, Rabbani H, et al. Comprehensive study of retinal vessel classification methods in fundus images. J Med Signals Sens. 2017:7:59-70

67. Haddock LJ, Kim DY, Mukai S. Simple, inexpensive technique for highquality smartphone fundus photography in human and animal eyes. J Ophthalmol. 2013;2013:1-6.

68. Sorsby A. Vital staining of the retina: preliminary clinical note. $\mathrm{Br} J$ Ophthalmol. 1939;23:20-4.

69. Fayed A, Fawzi A: OCTA vs. Dye: The Pros and Cons. Rev Ophthalmol 2019

70. Spaide RF, Klancnik JM. Retinal vascular layers imaged by fluorescein angiography and optical coherence tomography angiography. JAMA Ophthalmol. 2015;133:45-50.

71. Chen C-L, Wang RK. Optical coherence tomography based angiography [Invited]. Biomed Opt Express. 2017;8:1056-82.

72. Shin $\mathrm{YU}$, Lee DE, Kang MH, et al. Optical coherence tomography angiography analysis of changes in the retina and the choroid after haemodialysis. Sci Rep. 2018;8:17184

73. de Carlo TE, Romano A. A review of optical coherence tomography angiography (OCTA). Int J Retin Vitr. 2015;1:5.

74. Wylęgała A. Principles of OCTA and applications in clinical neurology. Curr Neurol Neurosci Rep. 2018;18(96):1-10.

75. Arthur E, Elsner AE, Sapoznik KA, et al. Distances from capillaries to arterioles or venules measured using OCTA and AOSLO. Investig Ophthalmol Vis Sci. 2019;60:1833-44.

76. Rocholz R, Corvi F, Weichsel J, et al. OCT Angiography (OCTA) in Retinal Diagnostics. High Resolution Imaging in Microscopy and Ophthalmology. Cham: Springer International Publishing; 2019; pp. 135-160

77. Li XX, Wu W, Zhou H, et al. A quantitative comparison of five optical coherence tomography angiography systems in clinical performance. Int J Ophthalmol. 2018;11(11):1784-95.

78. Jia Y, Tan O, Tokayer J, et al. Split-spectrum amplitude-decorrelation angiography with optical coherence tomography. Opt Express. 2012;20(4):4710

79. Turgut B. Optical coherence tomography angiography-a general view. Eur Ophthalmic Rev. 2016;10:39-42.

80. Kashani AH, Chen C-L, Gahm JK, et al. Optical coherence tomography angiography: a comprehensive review of current methods and clinical applications. Prog Retin Eye Res. 2017;60:66-100.

81. Toto L, Borrelli E, Mastropasqua R, et al. Association between outer retinal alterations and microvascular changes in intermediate stage age-related macular degeneration: an optical coherence tomography angiography study. Br J Ophthalmol. 2017;101:774-9.

82. Park J, Yongjoo K. Visualisation of 3D microcirculation of rodents retinas for studies of critical illness using OCTA. Crit Care Med. 2016:44(12):1455

83. Liu X, Kale AU, Capewell N, et al. Optical coherence tomography (OCT) in unconscious and systemically unwell patients using a mobile OCT device: a pilot study. BMJ Open. 2019;9:e030882.

84. Tan ACS. An overview of the clinical applications of optical coherence tomography angiography. Eye (Lond). 2018;32:262-86.

85. Arya M, Rashad R, Sorour O, et al. Optical coherence tomography angiography (OCTA) flow speed mapping technology for retinal diseases. Expert Rev Med Devices. 2018;15(12):875-82.

86. Borrelli E, Sadda SVR, Uji A, et al. Pearls and pitfalls of optical coherence tomography angiography imaging: a review. Ophthalmol Ther. 2019;8(2):215-26

87. Coscas F, Sellam A, Glacet-Bernard A, et al. Normative data for vascular density in superficial and deep capillary plexuses of healthy adults assessed by optical coherence tomography angiography. Investig Ophthalmol Vis Sci. 2016;57:OCT211-23.

88. Fernández-Vigo Jl, Kudsieh B, Shi H, et al. Normative database of peripapillary vessel density measured by optical coherence tomography angiography and correlation study. Curr Eye Res. 2020;45(11):1430-7.

89. Sato R, Kunikata H, Asano T, et al. Quantitative analysis of the macula with optical coherence tomography angiography in normal Japanese subjects: the Taiwa study. Sci Rep. 2019;9:1-11.

90. Fernández-Vigo Jl, Kudsieh B, Shi H, et al. Normative database and determinants of macular vessel density measured by optical coherence tomography angiography. Clin Exp Ophthalmol. 2020b;48(1):44-52.
91. Mihailovic N, Brand C, Lahme L, et al. Repeatability, reproducibility and agreement of foveal avascular zone measurements using three different optical coherence tomography angiography devices. PLoS ONE. 2018;13(10):e0206045.

92. Pappelis K, Jansonius NM. Quantification and repeatability of vessel density and flux as assessed by optical coherence tomography angiography. TransI Vis Sci Technol. 2019;8(3):3.

93. Lee $M-W$, Kim K-M, Lim H-B, et al. Repeatability of vessel density measurements using optical coherence tomography angiography in retinal diseases. Br J Ophthalmol. 2019;103:704-10.

94. Frost $\mathrm{S}$, Brown M, Stirling V, et al. Utility of ward-based retinal photography in stroke patients. J Stroke Cerebrovasc Dis. 2017;26:600-7.

95. Kwapong WR, Ye H, Peng C, et al. Retinal microvascular impairment in the early stages of parkinson's disease. Investig Opthalmol Vis Sci. 2018;59:4115-22.

96. Lad EM, Mukherjee D, Stinnett SS, et al. Evaluation of inner retinal layers as biomarkers in mild cognitive impairment to moderate Alzheimer's disease. PLoS ONE. 2018;13:e0192646.

97. Querques G, Borrelli E, Sacconi R, et al. Functional and morphological changes of the retinal vessels in Alzheimer's disease and mild cognitive impairment. Sci Rep. 2019;9:63.

98. Kersten HM. Optical coherence tomography findings in Huntington's disease: a potential biomarker of disease progression. J Neurol. 2015;262:2457-65.

99. Sweeney MD, Kisler K, Montagne A, et al. The role of brain vasculature in neurodegenerative disorders. Nat Neurosci. 2018;21:1318-31.

100. Pandharipande P. Long-term cognitive impairment after critical illness: commentary. J Pain Symptom Manage. 2014;369(14):1306-16.

101. Danesh-Meyer HV. Reduction of optic nerve fibers in patients with Alzheimer disease identified by laser imaging. Neurology. 2006;67:1852-4.

102. Jiang H, Liu Y, Wei Y, et al. Impaired retinal microcirculation in patients with Alzheimer's disease. PLoS ONE. 2018;13:e0192154.

103. Bulut M, Kurtuluş F, Gözkaya O, et al. Evaluation of optical coherence tomography angiographic findings in Alzheimer's type dementia. Br J Ophthalmol. 2018;102:233-7.

104. Zhang YS, Zhou N, Knoll BM, et al. Parafoveal vessel loss and correlation between peripapillary vessel density and cognitive performance in amnestic mild cognitive impairment and early Alzheimer's Disease on optical coherence tomography angiography. PLOS ONE. 2019;14:e0214685.

105. Kwa VIH. Retinal arterial changes correlate with cerebral small-vessel disease. Neurology. 2002;59:1536-40.

106. Eriksson S, Nilsson J, Sturesson C. Non-invasive imaging of microcirculation: a technology review. Med Devices (Auckl). 2014;7:445-52.

107. De Backer D, Creteur J. Microvascular blood flow is altered in patients with sepsis. Am J Respir Crit Care Med. 2002;166:98-104.

108. Kanoore Edul VS, Enrico C, Laviolle B, et al. Quantitative assessment of the microcirculation in healthy volunteers and in patients with septic shock. Crit Care Med. 2012;40:1443-8.

109. Ince C, Boerma EC, Cecconi M, et al. Second consensus on the assessment of sublingual microcirculation in critically ill patients: results from a task force of the European Society of Intensive Care Medicine. Intensive Care Med. 2018:44(3):281-99.

110. Sallisalmi M, Oksala N, Pettilä V, et al. Evaluation of sublingual microcirculatory blood flow in the critically ill. Acta Anaesthesiol Scand. 2012;56:298-306.

111. Aykut G, Veenstra G, Scorcella C, et al. Cytocam-IDF (incident dark field illumination) imaging for bedside monitoring of the microcirculation. Intensive Care Med Exp. 2015;3:1-10.

112. Hessler M, Arnemann PH, Zamit F, et al. Monitoring of conjunctival microcirculation reflects sublingual microcirculation in ovine septic and hemorrhagic shock. Shock. 2019;51:479-86.

113. Pranskunas A, Pilvinis V, Dambrauskas Z, et al. Early course of microcirculatory perfusion in eye and digestive tract during hypodynamic sepsis. Crit Care. 2012;16:R83.

114. Terheyden $\mathrm{JH}$. Impaired retinal capillary perfusion assessed by optical coherence tomography angiography in patients with recent systemic hypertensive crisis. Invest Ophthalmol Vis Sci. 2019;60:4573.

115. Alnawaiseh M. Evaluation of ocular perfusion in patients with atrial fibrillation using optical coherence tomography angiography. Invest Ophthalmol Vis Sci. 2019;60:4570. 
116. Arnould L, Guenancia C. The EYE-MI pilot study: a prospective acute coronary syndrome cohort evaluated with retinal optical coherence tomography angiography. Investig Opthalmol Vis Sci. 2018;59:4299.

117. Wang J, Jiang J, Zhang Y, et al. Retinal and choroidal vascular changes in coronary heart disease: an optical coherence tomography angiography study. Biomed Opt Express. 2019;10:1532

118. Kılınç Hekimsoy H, Şekeroğlu MA, Koçer AM, et al. Analysis of retinal and choroidal microvasculature in systemic sclerosis: an optical coherence tomography angiography study. Eye. 2020;34:763-70.

119. Mor G. Inflammation and pregnancy: the role of the immune system at the implantation site. Ann NY Acad Sci. 2011;1221:80-7.

120. Lin BR. Characterizing changes in retinal perfusion in high risk pregnancies with optical coherence tomography angiography. Invest Ophthalmol Vis Sci. 2019;60:4566.

121. Moshiri Y, Legocki AT, Zhou K, et al. Handheld swept-source optical coherence tomography with angiography in awake premature neonates. Quant Imaging Med Surg. 2019;9:1495-502.

122. Zadeh JK, Ruemmler R, Hartmann EK, et al. Responses of retinal arterioles and ciliary arteries in pigs with acute respiratory distress syndrome (ARDS). Exp Eye Res. 2019;184:152-61.

123. Alnawaiseh $M$, Ertmer $C$, Seidel L, et al. Feasibility of optical coherence tomography angiography to assess changes in retinal microcirculation in ovine haemorrhagic shock. Crit Care. 2018;22:138.

124. Park J. Microcirculatory alterations in hemorrhagic shock and sepsis with optical coherence tomography. Crit Care Med. 2016:44:1424.

125. Erikson K, Liisanantti JH, Hautala N, et al. Retinal arterial blood flow and retinal changes in patients with sepsis: preliminary study using fluorescein angiography. Crit Care. 2017;21:86

126. Simkiene J, Pranskuniene $Z$, Patasius $M$ et al.: Alterations of retinal vessels in patients with sepsis. J Clin Monit Comput 2019; 1-6

127. Ong Y-T, De Silva DA, Cheung CY, et al. Microvascular structure and network in the retina of patients with ischemic stroke. Stroke. 2013:44:2121-7.

128. Lee CW, Cheng HC, Chang FC, et al. Optical coherence tomography angiography evaluation of retinal microvasculature before and after carotid angioplasty and stenting. Sci Rep. 2019;9:14755.

129. Lahme L, Mihailovic N. Evaluation of ocular perfusion in Alzheimer's Disease using optical coherence tomography angiography. Invest Ophthalmol Vis Sci. 2019;60:4564.
130. Abraham A Guo X Retinal microvascular health and what it tells us about cognitive function: the eye determinants of cognition (EyeDOC) study. Invest Ophthalmol Vis Sci. 2019;60:4561.

131. Wang L, Kwakyi O, Nguyen J, et al. Microvascular blood flow velocities measured with a retinal function imager: inter-eye correlations in healthy controls and an exploration in multiple sclerosis. Eye Vis. 2018:5:29.

132. Yilmaz H, Ersoy A, Icel E. Assessments of vessel density and foveal avascular zone metrics in multiple sclerosis: an optical coherence tomography angiography study. Eye. 2020;34:771-8.

133. Lanzillo R, Cennamo G, Criscuolo C. Optical coherence tomography angiography retinal vascular network assessment in multiple sclerosis. Mult Scler J. 2018:24:1706-14.

134. Beare NAV, Harding SP. Perfusion abnormalities in children with cerebral malaria and malarial retinopathy. J Infect Dis. 2009;199:263-71.

135. Dallorto $L$, Lavia $C$, Jeannerot $A L$, et al.: Retinal microvasculature in pituitary adenoma patients: is optical coherence tomography angiography useful? Acta Ophthalmol 2019; 1-8

136. Suzuki ACF, Zacharias LC, Preti RC, et al. Circumpapillary and macular vessel density assessment by optical coherence tomography angiography in eyes with temporal hemianopia from chiasmal compression. Correlation with retinal neural and visual field loss. Eye. 2020;34:695-703.

137. Lee G-I, Park K-A, Oh SY, et al. Parafoveal and peripapillary perfusion predict visual field recovery in chiasmal compression due to pituitary tumors. J Clin Med. 2020;9:697.

\section{Publisher's Note}

Springer Nature remains neutral with regard to jurisdictional claims in published maps and institutional affiliations.

\section{Submit your manuscript to a SpringerOpen ${ }^{\circ}$ journal and benefit from:}

- Convenient online submission

- Rigorous peer review

- Open access: articles freely available online

- High visibility within the field

- Retaining the copyright to your article

Submit your next manuscript at springeropen.com 